\title{
Light mirror reflection and orientational irradiation automatic tracking control model \\ Shuying Wang ${ }^{{ }^{1}}{ }$, Zhanmin Yang $^{2}$,Yi Wang ${ }^{3}$ \\ 1,2 Nanjing Institute of Industry Technology, Nanjing, China; \\ ${ }^{3}$ School of computer science and technology ,Nanjing University of Science and Technology, Nanjing, China
}

* ${ }^{1}$ E-mail: 1972677541@qq.com

Keywords: Mirror reflection, Orientational irradation, Automatic tracking, Control model.

\begin{abstract}
On the basis of mirror reflection characteristics, the article analyzes the relation between the space plane that rotates about a point and reflective light change, designs mirror reflection and orientational irradiation automatic tracking control model, establishes signal conversion equation, drive speed selection equation, orthogonal axis rotation vector equation and reflection mirror rotation equations, sets the functional relations among the light incident angle, reflective angle, signal colletion and speed rotation as a list. Then, in combination with the directional reflection characteristics of the sunlight, the use method of the model are presented.
\end{abstract}

\section{Introduction}

People who live in the room on the back of the building can't see the sun all the year, which affects their health, the cold wet goods on the back of granary or warehouse are prone to become moldy. Due to lack of sunlight, the trees and flowers on the back of the building are withered. If the extra sunlight can be "transferred" to the area without sunlight, it will greatly improve the life of building and space utilization. To solve the problem, this article is to design mirror reflection and orientational irradiation automatic tracking control device.Provided that the device is installed in the area exposed to the sunshine near the building, the light can be automatically reflected to its shadow area with the reflective angle domain between $0^{\circ}-180^{\circ}$, and by thedirection of orientator the light is always reflected to the same place. This device is simple in structure, easily produced and installed with low cost and strong adaptability. It is based on the principle of rotation of the plane pointing, mirror reflective principle and the principle of one-way rectangular cylinder. This device use SCM to change the photoelectric signal into a driving signal to run. Through the two-dimensional rotation of the Reflective mirror, the reflected light is orientated to one direction. With a clock control function, it can let the system automatically return or evade bad weather so as to prolong the service life and reduce energy consumption.

\section{Reflective mirror}

In the space rectangular coordinate system $O-x y z$ of figure. $1, P\left(x_{0}, y_{0}, z_{0}\right)$ is a fixed-point on the fixed straight line $\bar{L}$ through the origin, $L$ is a straight line through the origin, $Q(x, y, z)$ is a fixed point on $L$, the direction angle of the vector $\overrightarrow{O Q}, \overrightarrow{O P}$ is $\alpha, \beta, \gamma$ and $\alpha_{0}, \beta_{0}, \gamma_{0}$ respectively. The equation of the plane $\Pi$,which through the origin $O$ with the normal vector of the $\overrightarrow{O Q}$, is

$$
x \cos \alpha+y \cos \beta+z \cos \gamma=0^{[1]} \text {. }
$$

On the plane $Q O Z$, axial symmetry straight line about the line $L$ by shaft $O Z$ is $L_{0}$.With a point

$P$ and the normal vector of $\overrightarrow{O P}$, the equation of plane $\amalg$ is

$$
x \cos \alpha_{0}+y \cos \beta_{0}+z \cos \gamma_{0}-\left(x_{0} \cos \alpha_{0}+y_{0} \cos \beta_{0}+z_{0} \cos \gamma_{0}\right)=0
$$

The angle between the plane $y O P$ and $y O Q$ through the coordinate axis $O y$ and coordinate plane $y O z$ is $\phi_{1}=\angle P_{1} O Z, \varphi_{1}=\angle Q_{1} O Z$ respectively, and the angle between the plane $x O P, x O Q$ through the 
coordinate axis $O x$ and coordinate plane $x O z$ is $\phi_{2}=\angle P_{2} O Z, \varphi_{2}=\angle Q_{2} O Z$ respectively, and there are $\tan \varphi_{1}=\cos \beta / \cos \gamma, \tan \varphi_{2}=\cos \alpha / \cos \gamma, \tan \phi_{1}=\cos \beta_{0} / \cos \gamma_{0}, \tan \phi_{2}=\cos \alpha_{0} / \cos \gamma_{0}$, with the result that direction cosine of the normal vector $\overrightarrow{O Q}, \overrightarrow{O P}$ of plane $\Pi, \amalg$ are as follows

$$
\begin{aligned}
& \cos \alpha=\frac{ \pm \tan \varphi_{1}}{\mu_{1}}, \cos \beta=\frac{ \pm \tan \varphi_{2}}{\mu_{1}}, \cos \gamma=\frac{ \pm \sqrt{\mu_{1}^{2}-\tan ^{2} \varphi_{1}-\tan ^{2} \varphi_{2}}}{\mu_{1}} ; \\
& \cos \alpha_{0}=\frac{ \pm \tan \phi_{1}}{\mu_{2}}, \cos \beta_{0}=\frac{ \pm \tan \phi_{2}}{\mu_{2}}, \cos \gamma_{0}=\frac{ \pm \sqrt{\mu_{2}^{2}-\tan ^{2} \phi_{1}-\tan ^{2} \phi_{2}}}{\mu_{2}} ; \\
& \left(\mu_{1}=\sqrt{\sec ^{2} \varphi_{1} \sec ^{2} \varphi_{2}+\tan ^{2} \varphi_{1} \tan ^{2} \varphi_{2}} \quad, \quad \mu_{2}=\sqrt{\sec ^{2} \phi_{1} \sec ^{2} \phi_{2}+\tan ^{2} \phi_{1} \tan ^{2} \phi_{2}}\right)
\end{aligned}
$$

By connecting two points $Q, P$ (figure. 2), $R$ is a point on the line $Q P, O R$ is the bisector of $\angle P O Q$, the angle between plane $x O R$ and coordinate surface $x O z$ is $\tau_{1}=\angle R_{1} O Z$ and angle between plane $y O R$ and coordinate plane $y O z$ is $\tau_{2}=\angle R_{2} O Z$, there are

$$
\tau_{1}=\frac{\phi_{1}+\varphi_{1}}{2}, \tau_{2}=\frac{\phi_{2}+\varphi_{2}}{2}
$$

Plane with the normal vector $\overrightarrow{O R}$ through the origin is $\Pi_{2}$, through a coordinate axis oy with the normal vector $\left(\sin \tau_{1}, 0, \cos \tau_{1}\right)^{[2]}$, the plane is $\Pi_{1}$, the line on the plane $\Pi_{1}$ through the origin and perpendicular to the axis $O y$ is $L_{1}$.

Given that $\vec{n}=\left(\alpha_{1}(t), \beta_{1}(t), \gamma_{1}(t)\right)$ is motion vector (t as parameter), through the origin and $\vec{n}$ is the normal vector, the equation of plane $\Sigma$ is

$$
x \cos \alpha_{1}(t)+y \cos \beta_{1}(t)+z \cos \gamma_{1}(t)=0
$$

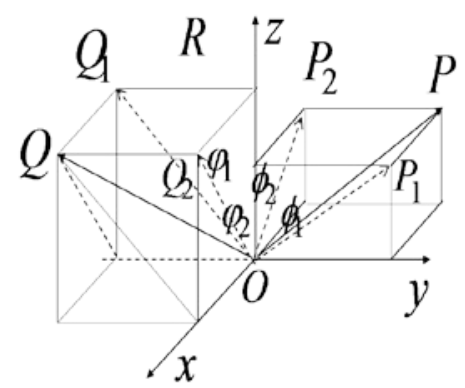

Fig.1.The vector and coordinate plane angle.

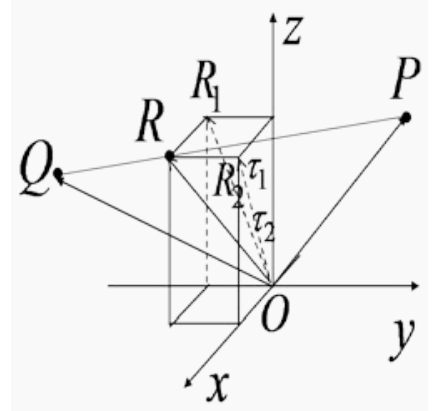

Fig.2.The reflected light angle.

When $t=0,1,2$, plane $\Sigma$ is $\Pi_{0}$ (equation $z=0$ ), $\Pi_{1}$ and $\Pi_{2}$ respectively. Plane $\Sigma$ coincides with the plane $\Pi_{1}$ after the plane $\Pi_{0}$ rotates $\tau_{1}$ around the axis $O y, \tau_{1}=\tau_{1}(t),(0 \leq t \leq 1)$. Angle $\tau_{1}$ is called angle in the zonal direction of plane $\Sigma$ and $O y$ is called the zonal shaft; When plane $\Sigma$ coincides with the plane ${ }_{\Pi_{2}}$ after plane $\Pi_{1}$ rotates $\tau_{2}$ around the straight line $L_{1} \cdot \tau_{2}=\tau_{2}(t),(1<t \leq 2), \tau_{2}$ is called angle in the zonal direction of plane $\Sigma ; L_{1}$ is called the warp shaft. The movement of the plane $\Sigma$ turning around the origin from the $\Pi_{0}$ to $\Pi_{1}$ to $\Pi_{2}$ is known as the plane $\Sigma$ orthogonal independence movement, with rotation vector as $\vec{\tau}=\left(\tau_{1}, \tau_{2}\right)$.If a straight line of $L$ represents a light that luminous body irradiates toward origin, three coordinate surfaces are its incident plane surface (light surface) ${ }^{[3]} ; \alpha, \beta, \gamma$, as direction angle of light $L$, are the angles of incidence light on the surface of $y O z, z O x, x O y$ respectively and the vertical plane of $L$ at the origin is the plane $\Pi$ in equation (1). If the plane $\Pi_{2}$ has the function of reflecting light, Line $L$ as the incident light, the reflective light $l_{2}$ and linear $\bar{L}$ overlap. So, plane $\Pi_{2}$ is called reflective mirror; vertical plane of the reflected light $l_{2}$ is the plane ${ }_{\amalg}$ in equation (2), which is called target straight plane, and straight line $\bar{L}$ is called orientational line. If plane $\Sigma$ is reflective mirror, the reflective light $l$ of the incident light ${ }_{L}$ at the origin complies with the direction 
of $\Sigma$. When $t=0, l_{0}$, reflective light $l_{0}$ of $L$ does not coincide with orientational line $\bar{L}$. When $t=2, l_{2}$, reflective light $l_{2}$ of $L$ coincides with orientational line $\bar{L}$. Then the reflective mirror ${ }_{\Pi_{2}}$ is called target mirror and rotating reflective mirror $\Sigma$ is called tracking mirror. After tracking mirror $\Sigma$ overlap with the target mirror $\Pi_{2}$, the luminous body moves and direction angle $(\alpha(t), \beta(t), \gamma(t))$ of the light $L$ continue to change with $t(t>2)$ and target mirror $\Pi_{2}$ will continue rotate around the origin with tracking mirror $\Sigma$ just consistent with the motion of plane $\Pi_{2}$. The process of tracking mirror $\Sigma$ from $\Pi_{0}$ to $\Pi_{2}$ is the fast track, afterwards the movement process consistent with $\Pi_{2}$ is synchronous tracking.

\section{Reflective rotatation device}

Component combination of figure3, is called orthogonal rotation mechanism. Among them, the rectangular plane $A B C D$ is installed inside a rectangular plane frame so $E_{1}, F_{1}, G_{1}, H_{1}$, in turn, is the midpoint of the border $A_{1} B_{1}, B_{1} C_{1}, C_{1} D_{1}, D_{1} A_{1}$ around the $A_{1} B_{1} C_{1} D_{1}$, a vertical axis $G_{1} W_{1}, H_{1} W_{2}$ of $A_{1} D_{1}$ fixed in $G_{1}, H_{1}$, two axis line $G_{1} W_{1}, H_{1} W_{2}$ in the same line, two axis outer end respectively fixed to the bracket to the $f$ ront end of the $\Lambda_{1}, \Lambda_{2}$. If motor $M_{1}$ is fixed on the bracket $\Lambda_{2}$ and motor $M_{1}$ is connected to the shaft $H_{1} W_{2}$. Rotation of the motor $M_{1}$ can drive the rectangular frame $A_{1} B_{1} C_{1} D_{1}$ to ratate around the axis $G_{1} H_{1} \cdot E, F$ is the midpoint of two parallel sides $A B, C D$ on the plane $A B C D$ respectively. An axis $E_{1} E, F_{1} F$ perpendicular to $A B$ is fixed at $E, F$ respectively, both the outer ends of the two shafts $E_{1} E, F_{1} F$ are respectively fixed in the bearing of $E_{1}, F_{1}$ on the frame $A_{1} B_{1} C_{1} D_{1}$. Two axises in the same line, in the $E_{1}$ of the framework $A B$ motor $M_{2}$ is equipped and the motor $M_{2}$ is connected with the shaft $E_{1} E$ so motor $M_{2}$ can drive plane $A B C D$ to rotate around the axis $E_{1} E$.

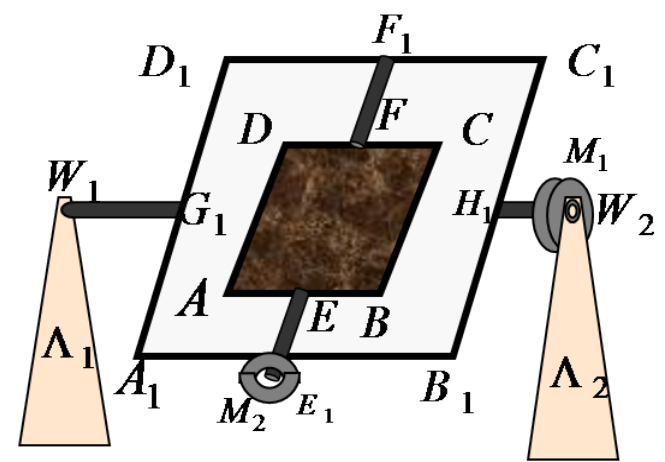

Fig.3.Independent rotating mechanism.

If bracket $\Lambda_{1}, \Lambda_{2}$ is fixed bracket $\Lambda_{1}, \Lambda_{2}$ motionless, in the rotating mechanism coordinate system $O-x y z$ is set in figure 1,2 , and the center of the rectangular plane frame of $A_{1} B_{1} C_{1} D_{1}$ is set as the origin $O$ so axis ${ }_{O y^{+}}$coincides with the axis $W_{1} W_{2}$, shaft $\mathrm{Ox}^{+}, \mathrm{Oy}^{+}$level, $\mathrm{Oz}^{+}$vertical. When the $A B C D$ and $A_{1} B_{1} C_{1} D_{1}$ coincides with coordinate plane ${ }^{x O y}$, plane $A B C D$ is $\Pi_{0}$, axis $E_{1} F_{1}$ on axis $O x$. Start the motor $M_{1}$ to let the $A B C D$ rotate the angle $\tau_{1}$ with $A_{1} B_{1} C_{1} D_{1}$, then close it. Then $A_{1} B_{1} C_{1} D_{1}$ is fixed, the plane of rectangle $A_{1} B_{1} C_{1} D_{1}$ as the plane $\Pi_{1}$. axis $E_{1} F_{1}$ coincident with the straight line $L_{1}$. Afterwards, start the motor $M_{2}$ to make the $A B C D$ to rotate the angle $\tau_{2}$ around the axis of $E_{1} F_{1}$ then close it, $A B C D$ as the plane $_{\Pi_{2}}$. In the process of operation, $A B C D$ is tracking plane $\Sigma$. Given that the angular velocity of the motor $M_{1}$ is $\varpi_{11}$, angular velocity of axis $W_{1} W_{2}$ is $\omega_{11}=k_{1} \varpi_{11}$ and angular velocity of motor $M_{2}$ is $\varpi_{21}$, angular velocity of axis $E_{1} F_{1} \omega_{21}=k_{2} \varpi_{21}, k_{1}, k_{2}$ the speed ratio of the motor $M_{1}, M_{2}$ and the rotating shaft $_{W_{1} W_{2}}, E_{1} F_{1}$ respectively. Rotation vector is:

$$
\vec{\tau}=\left(\tau_{1}, \tau_{2}\right)=\left(\omega_{11}\left(t_{1}-t_{0}\right), \omega_{21}\left(t_{2}-t_{1}\right)\right)=\left(k_{1} \varpi_{11}\left(t_{1}-t_{0}\right), k_{2} \varpi_{21}\left(t_{2}-t_{1}\right)\right)
$$


Because the axis of $W_{1} W_{2}, E_{1} F_{1}$ is vertically independent, plane $A B C D$ rotating from $\Pi_{0}$ to $\Pi_{2}$ can start motor $M_{1}$ and $M_{2}$ at the same time so the fast track shortest time is $\max \left\{t_{1}-t_{0}, t_{2}-t_{1}\right\}$.

Plane $A B C D$ is a mirror made of reflective material. When reflective mirror $A B C D$ become tracking mirror $\Sigma$, orthogonal rotating mechanism with tracking mirror is a reflective rotation device.

Plane $A B C D$ is a mirror made of reflective material. When reflective mirror $A B C D$ become tracking mirror $\Sigma$, orthogonal rotating mechanism with tracking mirror is a reflective rotation device.

\section{Orientational receiver}

Orientational receiver is composed of two dimensional independent rotating stents and receiver as shown in figure 3 , the rectangle shape of the receiver is a empty box $A_{2} B_{2} C_{2} D_{2}-{ }_{3} B_{3} C_{3} D_{3}$ and rectangle is surrounded by six plane $A_{2} B_{2} C_{2} D_{2}, A_{3} B_{3} C_{3} D_{3}, A_{2} D_{2} D_{3} A_{3}, B_{2} C_{2} C_{3} B_{3}, A_{2} B_{2} B_{3} A_{3}, C_{2} D_{2} D_{3} C_{3}$, the underside $A_{2} B_{2} C_{2} D_{2}$ square that side length is $2 a$, four sides $A_{2} D_{2} D_{3} A_{3}, B_{2} C_{2} C_{3} B_{3}, A_{2} B_{2} B_{3} A_{3}, C_{2} D_{2} D_{3} C_{3}$ rectangle that wide is $2 a$ and length is $h(h>2 a)$. The five surfaces are made of opaque material sheet, outer side of each sheet is coated with a layer of insulating material and the inner side of each one with uniform photosensitive materials (or photosensitive components). Photosensitive material irradiated by light can send induction signal (current) ${ }^{[4]}$, the intersecting lines between adjacent two pieces of sheet with insulation materials, the independent light signal sheet called light sensor, with $G_{i}(i=1,2 \cdots, 5)$, in turn to show the optical sensor on the plane $A_{2} B_{2} C_{2} D_{2}, A_{2} B_{2} B_{3} A_{3}, B_{2} C_{2} C_{3} B_{3}, C_{2} D_{2} D_{3} C_{3}, A_{2} D_{2} D_{3} A_{3}$. Each light sensor has separate wires to output signal, the upside $A_{3} B_{3} C_{3} D_{3}$ square which side length is $2 a$, named as the light incident port.

Receivers with rectangle box structure to reflect the light angle, which makes use of the parallel, orthogonal, shade properties in the plane of the wall, mainly include: avoiding light, when the illuminant below the plane of the upside $A_{3} B_{3} C_{3} D_{3}$, light can't irradiate into the receiver and the light sensors can't send signal; target tracking, when the light irradiates, all parallel to the side, i.e, the light irridates on the underside vertically, only light sensor $G_{1}$ has signal sent, which shows that synchronizes with the target; one-way, because of the block, the light on the same side of two parallel limited opaque plane, only one of them is exposed to it. Given that the lining of receiver is orthogonal and parallel, each plane is a direction with the result that signal combination is the least. And the adjacent photoreceptors are mutually perpendicular, parallel light incident from the opening, so three of five light sensors are irradiated at most. The light sensors irradiated simultaneously are adjacent to each other, easy to process control.

When receiver is installed on the upper portion of the orthogonal rotational independent institutions zonal rotor $W_{3} W_{4}$, it can rotate around the zonal $W_{3} W_{4}$, also with the plane frame around the longitudinal axis $E_{3} F_{4}$. The receiver is adjusted when $\Lambda_{3}$ stent is fixed, which makes the normal in the bottom center point to any direction. Orientational methods are:

As for static orientation, when the receiver is set in the coordinate system ${ }_{O-x y z}$ in figure 1 , 2, the center of underside $\mathrm{A}_{2} \mathrm{~B}_{2} \mathrm{C}_{2} \mathrm{D}_{2}$ coincides with coordinate plane $x \mathrm{Oy}$ at the origin, incident mouth up. Side $A_{2} B_{2} B_{3} A_{3}$ is vertical to axis $O x$ and in one side the positive direction while side ${ }_{2} C_{2} C_{3} B_{3}$ is vertical to axis $O y$ and in the one side of positive direction. If light parallel to linear $L$ irradiates to the inner wall of the receiver plane, angle of incidence is equal to the acute angle or the sharp angle of line $L$ direction angle $\alpha, \beta, \gamma$. Given that the incident angle of light on the surface of the five light sensor is set as $G_{i}(i=1,2 \cdots, 5) 0 \leq \theta_{i}<\pi / 2(i=1,2 \cdots, 5)$ respectively, the equation is,

$$
\theta_{1}=\gamma, \theta_{2}=\pi-\alpha(\alpha \geq \pi / 2), \theta_{3}=\pi-\beta(\beta \geq \pi / 2), \theta_{4}=\alpha, \theta_{5}=\beta
$$

With the angle of light $L$ into the receiver, equation (7) has different value combination. Given that the light is irradiated or not, when the light sensor $G_{i}$ is irradiated, signal is set as $G_{i}$, otherwise, 
signal as $\overline{G_{i}}$, At the same time five light sensors $G_{i}$ form a signal combination. If $f\left(G_{i}\right)$ is set as the viscosity of signal combination, the relationship between $f\left(G_{i}\right)$ and light incident angle $\theta_{i}$ of $L$ is

$$
f\left(G_{1}, G_{2}, G_{3}, G_{4}, G_{5}\right)=\Phi\left(\theta_{1}, \theta_{2}, \theta_{3}, \theta_{4}, \theta_{5}\right)
$$

all relationships of (7) (8) are listed in table 1.

Table 1. The light angle and combination of the signal.

\begin{tabular}{|c|c|}
\hline$\Phi\left(\theta_{1}, \theta_{2}, \theta_{3}, \theta_{4}, \theta_{5}\right)$ & $f\left(G_{1}, G_{2}, G_{3}, G_{4}, G_{5}\right)$ \\
\hline$\theta_{i}<0$. & $\overline{G_{1}} \overline{G_{2}} \overline{G_{3} G_{4}} \overline{G_{5}}$. \\
\hline$\theta_{1}=0, \theta_{i} \neq 0, i \neq 1$. & $G_{1} \overline{G_{2}} \overline{G_{3} G_{4}} \overline{G_{5}}$. \\
\hline$\theta_{3,5}=\pi / 2$ & $\overline{G_{1}} G_{2} \overline{G_{3} G_{4} G_{5}}, G_{1} G_{2} \overline{G_{3} G_{4} G_{5}}, \overline{G_{1} G_{2} G_{3}} G_{4} \overline{G_{5}}, G_{1} \overline{G_{2} G_{3}} G_{4} \overline{G_{5}}$ \\
\hline$\theta_{2,4}=\pi / 2$ & $\overline{G_{1} G_{2}} G_{3} \overline{G_{4} G_{5}}, G_{1} \overline{G_{2}} G_{3} \overline{G_{4} G_{5}}, \overline{G_{1}} \overline{G_{2}} \overline{G_{3}} \overline{G_{4}} G_{5}, G_{1} \overline{G_{2} G_{3} \overline{G_{4}}} G_{5}$ \\
\hline $0<\theta_{i}<\pi / 2$ & $G_{1} G_{2} G_{3} G_{4} G_{5}, G_{1} G_{2} G_{3} G_{4} G_{5}, G_{1} G_{2} G_{3} G_{4} G_{5}, G_{1} G_{2} G_{3} G_{4} G_{5}$, \\
\hline & $G_{1} G_{2} G_{3} G_{4} G_{5}, G_{1} G_{2} G_{3} G_{4} G_{5}, G_{1} G_{2} G_{3} G_{4} G_{5}, G_{1} G_{2} G_{3} G_{4} G_{5}$. \\
\hline
\end{tabular}

In table 1 signal combination $G_{1} \overline{G_{2}} \overline{G_{3} G_{4} G_{5}}$ indicates that the optical sensor $G_{1}$ of the underside of the receiver $A_{2} B_{2} C_{2} D_{2}$, underside of the receiver is irradiated and four sides are not irradiated.

Dynamic orientation,In the static directional position, to make the underside of the receiver $\mathrm{A}_{2} \mathrm{~B}_{2} \mathrm{C}_{2} \mathrm{D}_{2}$ become light $L$ point-blank, we let receiver turn around the origin, the original normal $\mathrm{Oz}$ and light $L$ overlap, and rotate zonal rotor $W_{3} W_{4}$ of the receiver $\varphi_{1}$ and rotate wrap rotor $E_{3} F_{4} \varphi_{2}$. In the process of rotation, light $L$ changes with the incidence angle $\theta_{i}$ of two axises and the angle vector is $\vec{\varphi}=\left(\varphi_{1}, \varphi_{2}\right)$ with signal combinations $G_{1} \overline{G_{2}} \overline{G_{3}} \overline{G_{4}} \overline{G_{5}}$. By equation (8) the angle equation of the light surface into direct surface as follows $f\left(G_{i}\right)=\Phi\left(\theta_{i}\right)=\Phi(\alpha, \beta, \gamma)=g\left(\varphi_{1}, \varphi_{2}\right)$.

As for the orientational tracking of specular reflective light, orientational receiver $\Lambda_{3}$ is fixed on the same side of the reflective rotation mechanism and irridators, In the coordinate system $O-x y z$ of the reflective rotation mechanism, the underside $A_{2} B_{2} C_{2} D_{2}$ of the receiver is adjusted to parallel with the target plane ${ }_{U}$ with entrance port $A_{3} B_{3} C_{3} D_{3}$ towards the origin and the straight line $\bar{L}$ normal at the center of underside $A_{2} B_{2} C_{2} D_{2}$. The sides ${ }_{B_{2}} C_{2} C_{3} B_{3}, A_{2} D_{2} D_{3} A_{3}$ is perpendicular to $x O y$. When the reflective mirror $A B C D$ (tracking mirror $\Sigma$ ) is level, the incidence angle $\theta_{i}$ of the reflective light $l_{0}$ of light $L$ on the inner wall of directional receiver reflects the relationship between $l_{0}$ and $\bar{L}$. When $\theta_{1}=0, l_{0}$ directs target plane $\amalg$; when $\theta_{1} \neq 0$, target plane $\amalg$ is not a direct plane. To adjust the reflective mirror $A B C D$ to make the reflective light $l_{0}$ overlap with the target straight $\bar{L}$, we let the $A B C D$ turn around the origin to make the original normal $O z$ and linear $L_{1}$ overlap,i.e., $A B C D$ turning to the plane $\Pi_{2}$. And angle vector of $A B C D$ should be $\vec{\tau}=\left(\tau_{1}, \tau_{2}\right)$. Because the receiver is fixed, the direction angle $\alpha_{0}, \beta_{0}, \gamma_{0}$ is constant, by equation (3) (4) (8), we can get angle equations that reflective light becomes a orientational light as follows

$$
\Phi\left(\theta_{i}\right)=f\left(G_{i}\right)=\Phi(\alpha, \beta, \gamma)=g\left(\tau_{1}, \tau_{2}\right)
$$

\section{Drive controller}

Controllers are microprocessors made of electronic components and circuit boards or single chip microcomputer with auxiliary device. The input end of controller is connected with the output end of each photosensitive device of the signal receiver and the output end is connected with the motor $M_{1}, M_{2}$ of the reflective rotation mechanism. Motor $M_{1}, M_{2}$ can be set various speeds. Controller can convert electrical signals from the receiver to controllable signal to output so as to control the speed of 
motor $M_{1}, M_{2}$. If the speed vector of the motor is $V\left(v_{1}, v_{2}\right)$, the conversion between the speed vector and characterization value $f\left(G_{i}\right)$ of signal combination is

$$
V\left(v_{1}, v_{2}\right)=F\left(f\left(G_{1}, G_{2}, \cdots, G_{5}\right)\right)
$$

If the speed of the motor $M_{1}, M_{2}$ is set three levels as conventional speed $V_{0}\left(v_{10}, v_{20}\right)$, fast speed $V_{1}\left(v_{11}, v_{21}\right)$ and fast reverse speed $V_{2}\left(v_{12}, v_{22}\right)$ in the opposite direction, the speed of the motor $M_{1}$ is $v_{10}, v_{11}, v_{12}$ in turn and the speed of the motor $M_{2}$ is $v_{20}, v_{21}, v_{22}$ in turn ., When the reflective light on the receiver $G_{2} G_{3}$, motor is regarded as fast speed; when the reflective light on the receiver $G_{4} G_{5}$ motor fast reverse speed in the opposite direction and when the light is parallel to the side of the receiver, the motor conventional speed. In equation (10), the relationships of the combination between signal and motor speed motor speeds are given in table 2 .

Table 2. The combination and motor speed signal corresponding to the table

\begin{tabular}{|c|c|c|}
\hline$f\left(G_{1}, G_{2}, G_{3}, G_{4}, G_{5}\right)$ & $v_{1}$ & $v_{2}$ \\
\hline $\bar{G}_{1} G_{2} G_{3} G_{4} G_{5}$. & $\mathbf{O}$ & $\mathbf{O}$ \\
\hline$G_{1} \overline{G_{2}} \bar{G}_{3} G_{4} \bar{G}_{5}$. & $v_{10}$ & $v_{20}$ \\
\hline$\overline{G_{1}} G_{2} \overline{G_{3}} \bar{G}_{4} \bar{G}_{5}, G_{1} G_{2} \overline{G_{3} G_{4} G_{5}}$. & $v_{11}$ & $v_{20}$ \\
\hline$\overline{G_{1}} \overline{G_{2}} G_{3} \overline{G_{4}} \overline{G_{5}}, G_{1} \overline{G_{2}} G_{3} \overline{G_{4}} \bar{G}_{5}$. & $v_{10}$ & $v_{21}$ \\
\hline$\overline{G_{1}} \overline{G_{2}} \overline{G_{3}} G_{4} \overline{G_{5}}, G_{1} \overline{G_{2}} \overline{G_{3}} G_{4} \overline{G_{5}}$. & $v_{12}$ & $v_{20}$ \\
\hline$G_{1} G_{2} G_{3} G_{4} G_{5}, G_{1} G_{2} G_{3} G_{4} G_{5}$ & $v_{10}$ & $v_{22}$ \\
\hline$\overline{G_{1}} G_{2} G_{3} \overline{G_{4} G_{5}}, G_{1} G_{2} G_{3} \overline{G_{4} G_{5}}$. & $v_{11}$ & $v_{21}$ \\
\hline$\overline{G_{1}} \bar{G}_{2} G_{3} G_{4} \overline{G_{5}}, G_{1} \overline{G_{2}} G_{3} G_{4} \overline{G_{5}}$. & $v_{12}$ & $v_{21}$ \\
\hline$\overline{G_{1}} G_{2} G_{3} G_{4} G_{5}, G_{1} G_{2} G_{3} G_{4} G_{5}$. & $v_{11}$ & $v_{22}$ \\
\hline$\overline{G_{1}} G_{2} \overline{G_{3}} \bar{G}_{4} G_{5}, G_{1} G_{2} \bar{G}_{3} \bar{G}_{4} G_{5}$. & $v_{12}$ & $v_{22}$ \\
\hline
\end{tabular}

Table 3. Combined with motor speed signal corresponding to the table

\begin{tabular}{|c|c|c|c|c|c|c|c|c|}
\hline$\alpha_{0}(t)$ & $\beta_{0}(t)$ & $\gamma_{0}(t)$ & $\Phi\left(\theta_{1}, \theta_{2}, \theta_{3}, \theta_{4}, \theta_{5}\right)$ & $f\left(G_{1}, G_{2}, G_{3}, G_{4}, G_{5}\right)$ & $v_{1}$ & $v_{2}$ & $\tau_{1}$ & $\tau_{2}$ \\
\hline$\pi / 2$ & $\pi / 2$ & 0 & $\theta_{i}<0$ & $\overline{G_{1}} \overline{G_{2}} \overline{G_{3} G_{4}} \overline{G_{5}}$. & 0 & 0 & 0 & 0 \\
\hline$\alpha_{0}$ & $<\beta_{0}$ & $\gamma_{0}$ & $\theta_{1}=0, \theta_{i} \neq 0$ & $G_{1} \overline{G_{2}} \overline{G_{3} G_{4}} \overline{G_{5}}$. & $v_{10}$ & $v_{20}$ & $\omega_{10} t$ & $\omega_{20} t$ \\
\hline$<\alpha_{0}$ & $<\beta_{0}$ & $>\gamma_{0}$ & $\theta_{3,5}=\pi / 2, \theta_{2} \neq \pi / 2$ & $\overline{G_{1}} G_{2} \overline{G_{3}} \overline{G_{4}} \overline{G_{5}}, G_{1} G_{2} \overline{G_{3} G_{4} G_{5}}$. & $v_{11}$ & $v_{20}$ & $>0$ & 0 \\
\hline$>\alpha_{0}$ & $>\beta_{0}$ & $>\gamma_{0}$ & $\theta_{2,4}=\pi / 2, \theta_{3} \neq \pi / 2$ & $G_{1} G_{2} G_{3} G_{4} G_{5}, G_{1} G_{2} G_{3} G_{4} G_{5}$. & $v_{10}$ & $v_{21}$ & 0 & $<0$ \\
\hline$>\alpha_{0}$ & $>\beta_{0}$ & $<\gamma_{0}$ & $\theta_{3,5}=\pi / 2, \theta_{4} \neq \pi / 2$. & $\overline{G_{1} G_{2} G_{3}} G_{4} \overline{G_{5}}, G_{1} \overline{G_{2} G_{3}} G_{4} \overline{G_{5}}$. & $v_{12}$ & $v_{20}$ & $<0$ & 0 \\
\hline$>\alpha_{0}$ & $<\beta_{0}$ & $<\gamma_{0}$ & $\theta_{3,5}=\pi / 2, \theta_{5} \neq \pi / 2$ & $\overline{G_{1} G_{2} G_{3} G_{4}} G_{5}, G_{1} \overline{G_{2} G_{3} G_{4}} G_{5}$. & $v_{10}$ & $v_{22}$ & 0 & $>0$ \\
\hline$<\alpha_{0}$ & $<\beta_{0}$ & $>\gamma_{0}$ & $0<\theta_{1,2,3}<\pi / 2$ & $\overline{G_{1}} G_{2} G_{3} \overline{G_{4} G_{5}}, G_{1} G_{2} G_{3} \overline{G_{4} G_{5}}$. & $v_{11}$ & $v_{21}$ & $>0$ & $<0$ \\
\hline$>\alpha_{0}$ & $<\beta_{0}$ & $<\gamma_{0}$ & $0<\theta_{1,3,4}<\pi / 2$ & $\overline{G_{1} G_{2}} G_{3} G_{4} \overline{G_{5}}, G_{1} \overline{G_{2}} G_{3} G_{4} \overline{G_{5}}$. & $v_{12}$ & $v_{21}$ & $<0$ & $<0$ \\
\hline$>\alpha_{0}$ & $>\beta_{0}$ & $<\gamma_{0}$ & $0<\theta_{1,4,5}<\pi / 2$ & $\overline{G_{1}} G_{2} G_{3} G_{4} G_{5}, G_{1} \overline{G_{2}} \overline{G_{3}} G_{4} G_{5}$. & $v_{11}$ & $v_{22}$ & $<0$ & $>0$ \\
\hline$<\alpha_{0}$ & $>\beta_{0}$ & $>\gamma_{0}$ & $0<\theta_{1,2,5}<\pi / 2$ & $\overline{G_{1}} G_{2} \overline{G_{3}} \overline{G_{4}} G_{5}, G_{1} G_{2} \overline{G_{3}} \overline{G_{4}} G_{5}$ & $v_{12}$ & $v_{22}$ & $>0$ & $>0$ \\
\hline
\end{tabular}

\section{Sunlight reflective orientation tracking control}

The device made of reflective rotation mechanism, orientational receiver and drive controller is called the reflective light tracking system. The working process is as follows, If the signal combination $f\left(G_{i}\right)$ which is formed from the reflective light incident angle $\theta_{i}$ is processed by controller to send as instruction signal, drive motor receive instructions and set speed, and motor rotation is converted into the rotation reflective rotation mechanism axis, thus making the rotation of the shaft change direction of reflective mirror with the result that the reflective light adjusted by reflective mirror movement is consistent with the goal line. By equation (1) - (10), the orientational reflection is given as follows:

$$
\theta_{i} \stackrel{\Phi\left(\theta_{i}\right)}{\longrightarrow} G_{i}\left(\theta_{i}\right) \stackrel{f\left(G_{i}\right)}{\longrightarrow} V\left(v_{1}, v_{2}\right) \stackrel{K\left(v_{j}\right)}{\longrightarrow} \omega\left(\omega_{1 k}, \omega_{2 k}\right) \stackrel{\left(\phi_{1}, \phi_{2}\right)}{\longrightarrow} \vec{n}\left(\alpha_{1}, \beta_{1}, \gamma_{1}\right) \rightarrow \theta_{i}(\vec{n})
$$

Various relationships formed by type (11) are listed in table 3.

In table 3 , the $\omega_{10} t, \omega_{20} t$ are set as zonal angle and wrap angle that irradators move around the point on the sphere.Equation (11) and table 3 are used in the sunlight reflective orientation tracking 
control, easy to parameter determination, popularization and application. On the Earth's surface where reflective rotation device is fixed in the area with sunlight, zonal rotor $W_{1} W_{2}$ is set eastwestward and horizontal, tracking mirror up, and oriental receiver is fixed at the south of the reflective rotation device, with its open towards the tracking mirror center, bottom surface normal towards target. In the coordinate system $O-x y z$ of reflective tracking system, the sun as a particle, the sunlight is regarded as line $L$ so, that the sun rises from the east and set from the west and the season changes, is the Earth's rotation around the sun and the revolution movement ${ }^{[5]}$, which reflects linear $L$ synthesizes the warp movement and the zonal movement of the particle on the spherical surface, i.e., the warp angle and zonal angle change of dynamic linear $L$ around the origin to. The sun rise and fall speed is equal to the earth's rotation rate $\omega_{10}=2 \pi / T_{\beta}$, and speed of the sun seasonal movement is equal to the earth's orbital angular velocity $\left|\omega_{20}\right|=4 \gamma_{0} / T_{\alpha}\left(T_{\alpha}, T_{\beta}\right.$ earth's orbital period and rotation period respectively, $\gamma_{0}$ the angle between earth's equator and the ecliptic plane ${ }^{[6]}$ ).

Key point of system parameter is speed setting of the motor , so the method is:Synchronous tracking speed of the system, conventional motor velocity vector $V_{0}\left(v_{10}, v_{20}\right)=K\left(\omega_{10}, \omega_{20}\right),\left(\omega_{10}=2 \pi / T_{\beta}\right.$ as shaft angular velocity of rotation axis $W_{1} W_{2},\left|\omega_{20}\right|=4 \gamma_{0} / T_{\alpha}$ as the angular velocity of the shaft of rotation axis $\left.E_{1} F_{1}\right)$.

Fast tracking speed of the system, the fast tracking speed and fast reverse speed of the motor is $V_{1}\left(v_{11}, v_{21}\right), V_{2}\left(v_{12}, v_{22}\right)$, so, such factors as its friction, load, motor power should be considered to set based on equation (6). System initialization setting, due to the discontinuous appearance and the change in the weather, sunlight toward the ground is intermittent. To save energy consumption and increase system lifetime, the system only works when there is sunlight, otherwise, it stops work. The largest irradiating angle of two adjacent light is $180^{\circ}$ (morning and evening), the interval is a night when the weather is fine, while interval is not regular when the weather is not fine. In order to facilitate the job next time, clock function should be added in the control system and, after the continuous time $T_{0}$ without light irradiation, the system go back to the position at noon of the last working day.

\section{Conclusion}

Based on the plane and the normal characteristics, this paper analyses the movement principle of the reflective mirror around point, designs the orientational reflective tracking device, describes structure and function of the mirror rotation mechanism, the orientational receiver and drive controller, and establishes the models of reflective mirror rotation equation, light reflection signal sets, signal conversion equation, the velocity vector of the drive speed, and orthogonal axis rotation vector sets and so on. It gives the appropriate list of control relationship. Combined with the movement of the sun, the using method of the device is illustrated and the clock function is recommended to be adopted to improve the system initialization.

\section{Acknowledgements}

Nanjing Institute of Industry Technology scientific research fund key projects (No, kT-150501).China's ministry of education teaching vocational colleges informatization refers to appoint key subject (No,2013LX003)

\section{References}

[1] Guoquan Hu, geometry and algebra guidance, Beijing. science press, 2006

[2] WenMao Yang, QuanYing Li, Space analytic geometry, Wuhan: Wuhan university press, 2002.

[3] Shuying Wang, Solar Energy Automatic Tracking System Based on Plane Normal Vector Control Research,Switzerland: Advanced Material Research .Vol.748,PP:811-814,2013. 
[4] Shensheng Li, An introduction to solar energy heat utilization Beijing: Higher education press, 1989.

[5] Yi Su, Introduction to astronomy new, Beijing: science press , 2009.

[6] WeiXin Jiao, Hong Zou: Planetary science(Peking University press, China 2009) 